\title{
Application of Image Processing In E-Commerce
}

\section{LokeshVenkata Sai Mamidi, Pisupati Chaitanya, VikasUpadhyaya}

Abstract: The advancement and perpetual development in technology have made it possible to automate many processes. The proposed Algorithm in this research provides the framework to self-operate the process of quantifying the shoulder size of humans by taking the images of the user so that it can be utilized to find the shirt size of the human. The framework involves three important phases which are segmentation, edge detection, predicting shirt size. Since colour has no prominent role in measurement of size,otsu's binary thresholding for image segmentation is used in order to get binary image which separates foreground and background. Along with predictive analysis particularly regression is used as the groundwork to predict shirt size. The main application is in the apparel industry such as online shopping to automate the size detection for more expeditious results. And in the custom made apparel stitching, rather than approaching the seamster to take tape measurements our framework can be implemented therefore increasing the time efficiency.

Keywords:Image processing, application of data analysis, Otsu segmentation, measurement of a body part, Regression.

\section{INTRODUCTION}

In this research, we present a method for computing the measurement of the human upper body part and extract its size. The traditional methods to measure the size of the body part is either by approaching the seamster who uses a meter stick or measuring the size manually using tapeline. The proposed approach combines the power of computer vision and machine learning algorithms to get the desired output. In the initial phase, the algorithm acquires the image from the user and applies enhancement and noise removal techniques to improve the quality of an image. In the further phases, it applies the segmentation on an Image to calculate the pixel length of a shoulder and in the final phase, it predicts the actual size by using linear regression analysis. The proposed approach can find the size up to a minimal degree of error, and it can be improvised to predict the size with one Image, to reduce approximation error and to obtain better results we are providing three images taken from different distances as input.

Revised Manuscript Received on December 30, 2019.

* Correspondence Author

LokeshVenkata Sai Mamidi, (B Tech) UG Scholar, Batch (20162020), NIIT University,

Pisupati Chaitanya, B Tech) UG Scholar, Batch (2016-2020), NIIT University,

VikasUpadhyaya, B Tech) UG Scholar, Batch (2016-2020), NIIT University,

(C) The Authors. Published by Blue Eyes Intelligence Engineering and Sciences Publication (BEIESP). This is an open access article under the CC BY-NC-ND license (http://creativecommons.org/licenses/by-nc-nd/4.0/)

\section{LITERATURE REVIEW}

Liang Zhao and Chuck Thorpe[1] proposed an RCR approach to detect and identify the human body and its parts. This approach uses a predefined human model for detection \& model learning. The important aspect of this algorithm is that the algorithm is independent of how the initial contours are extracted from the image and it divides the initial contour to small segments and checks them one by one to check whether the contours are present in our human body model and confirms whether a human is detected using the decision rule repeatedly. This algorithm has some predefined conditions to Stop the loop: a) user can make a decision whether the image contains a person or not b) No more human body parts can be identified c) if no further improvement or changes can be made. This algorithm is Complex and hard to implement. Another researcher AnujMohan[2] proposed a method which uses classifier and combination algorithm which uses component and combination classifiers. The component classifiers detect components of the persons object i.e. heads, legs and arms separately. The combination classifier takes the input from the output of component classifiers and classifies the entire pattern under examination as a person or a nonperson. In this proposed approach, training each of the classifiers require huge dataset and also time-consuming. Other researchers YasirSalih and Aamir S. Malik[3] used a triangulation method of geometry to calculate the depth of field and geometry of the object in the $2 \mathrm{~d}$ image. This method can also be used for computing the actual size of the object in the scene as well as the distance between the different objects in the $2 \mathrm{~d}$ image using trigonometric relations. It requires inputs like Camera height, Camera pitch angle, Camera field of view. Since this method requires more parameters to be given by the user as compared to other algorithms, it is not user-friendly. other researchers J. C. S. Jacques, L. Dihl, C. R. Jung[4] focused on human upper body segmentation. They used a predefined face detection algorithm proposed by Viola and Jones as the groundwork to define search regions for the chest and shoulders. After detecting the face, comes the rough estimation of the skin pixels using RGB test. Based on the skin colour model, a region is defined based on the detected face that accommodates the upper body and arms. The chest of a person should be located right below the face of the person, and therefore a rectangular region is defined by a formula.

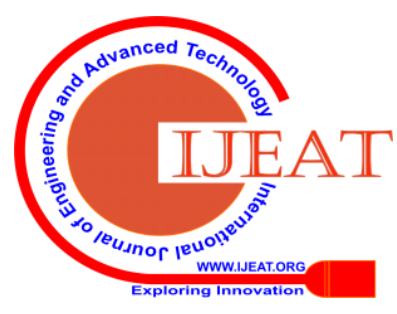




\section{Application of Image Processing In E-Commerce}

The upper corners of the boundary box of the chest are bound to be the person's shoulder, but due to the inaccuracy of the segmentation method, the points sometimes are not in their proper positions. To eliminate this error, they have used anthropometric parameters to refine the position of the shoulders. Another researchers MissHetal J. Vala, Prof. AsthaBaxi[5] discussed about Otsu's thresholding which is used to divide an image into different classes, in our case we are using this thresholding technique to classify the image as foreground and background classes in order to detect object this is achieved by automatically finding an optimal threshold based on the observed scattering of pixel values. The aim is to find the threshold where the sum of foreground and background spreads is at its minimum. In order to achieve this, it tries to maximize inter-class variance which is same as minimizing intraclass variance and is given by "equation (2.1)".

$$
\begin{aligned}
& \sigma_{b}^{2}(t)=\sigma^{2}-\sigma^{2}{ }_{\omega}(t) \\
& =\omega_{0}\left(\mu_{0}-\mu_{\mathrm{T}}\right)^{2}+\omega_{1}\left(\mu_{0}-\mu_{\mathrm{T}}\right)^{2} \\
& =\omega_{0}(\mathrm{t}) \omega_{1}(\mathrm{t})\left[\mu_{0}(\mathrm{t})-\mu_{1}(\mathrm{t})\right]^{2}
\end{aligned}
$$

Weights w0 and w1 are probabilities of the two classes and $\sigma^{2}{ }_{\omega}(t)$ is a weighted sum of variances of two classes $\sigma^{2} b(t)$ is inter-class variance while $\mu_{0}$ and $\mu_{1}$ are means. Due to its simple calculation and the fast result we are using this approach. Other researchers Bugao Xu, YueqiZhong[6] focused on Automatic segmentation and measurement of a scanned body part. In this procedure, two different methods are planned to find the crotch point for the situation of the non-contacted thigh and contacted thigh, respectively. Three different methods: minimum distance algorithm, minimum inclination angle algorithm, and directional neighbour identification algorithm are introduced to search the branching points. In the measurement procedure, a presorted circling method is designed for circumference quantification, and the basic principle of landmark acquisition has been discussed. These techniques are checked via testing over a different type of scanned model. The accurate and automatic locating of the wrist, ankle and knee contour will be harder than it appears to be. It also explains the fundamental measuring methods and gives a rough idea of the results of using these techniques in segmentation and measurement.

\section{PROPOSED METHODOLOGY}

As mentioned in "Fig. 3.1", we acquire images of the person from different distances $(75 \mathrm{~m}, 100 \mathrm{~m}, 150 \mathrm{~m})$ which are predefined, and give them as input to the system where the images are analyzed and proceed down to further phases. In this stage, we filter the images so as to reduce the noise which is possible to occur at the time of retrieval. Since we are calculating the size, colour has no prominent value for this system. Hence, these images are then converted into grayscale so as to decrease the complexity of the system. Later we have to extract contours and the first thing to be done in order to extract contours is binarization of an image which is done by thresholding method. We have opted Otsu's global thresholding method for it. After the segmentation, we use morphological operations to reduce the noise occurred in the previous stage. Later we find the contour of an image. Next step is to find the pixel length of the shoulder. Here we are finding the extreme points of the contour and calculating the distance between those points which will be discussed later. After getting the pixel length we need to find the actual length of shoulder. This will be done using regression analysis and then shoulder width is converted to shirt size.

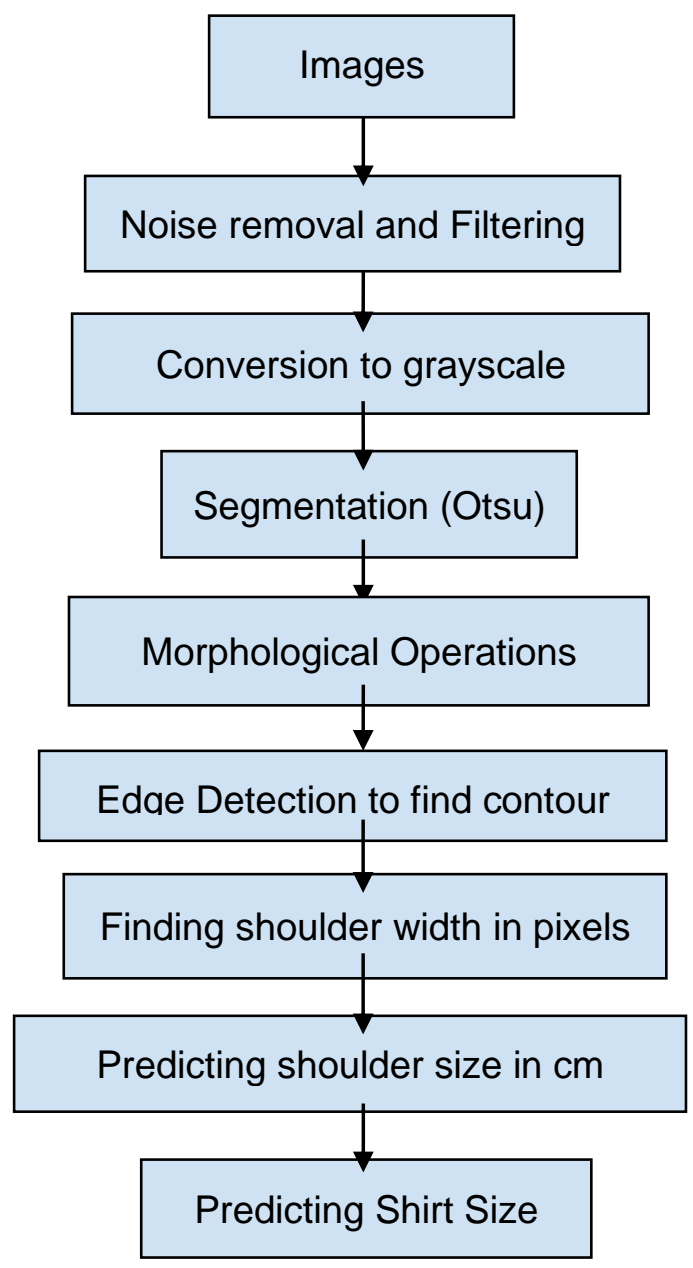

Fig. 3.1. Methodology

\section{RESULTS AND DISCUSSIONS}

\section{A. Image Acquisition \& Filtering}

As said earlier we have to provide three images as input captured from different distances $(75 \mathrm{~cm}, 100 \mathrm{~cm}, 150 \mathrm{~cm})$ and each image should be in the format as shown in "Fig.4.1". The image must have a plain background and the person should wear a plain shirt to reduce the noise. After acquiring the images, we apply Gaussian filters[7] on each image as it blurs the image, reducing the noise. 


\section{B. Segmentation \& Morphological operations}

Segmentation is the basic step for image processing. Since colour has no prominent role in measurement of size, we've done binary segmentation using Otsu global thresholdingmethod[5]. Binary images may contain numerous imperfections. Morphological operations[8] pursue the goal of removing these imperfections by accounting for the form and structure of the image. We have used morphological opening and closing operations. An opening operation can open up a gap between objects connected through a thin bridge of pixels. Closing can fill holes in the regions while maintaining the initial region sizes. "Fig. 4.2" will be the output after performing segmentation and morphological operations.

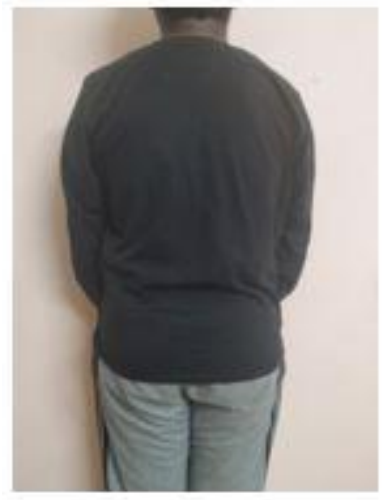

Fig. 4.1. Input Image

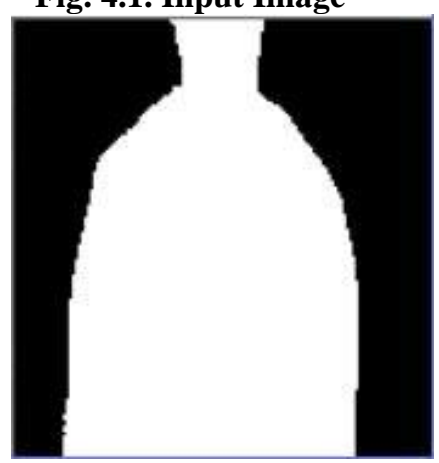

Fig. 4.2. Image after segmentation and Morphological operations

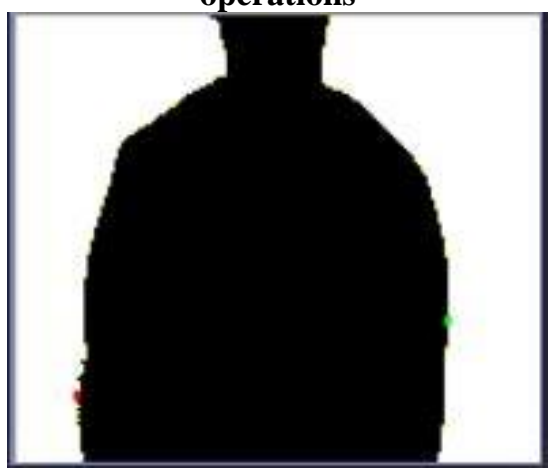

Fig. 4.3. Image after finding Extreme points (Red, Green)

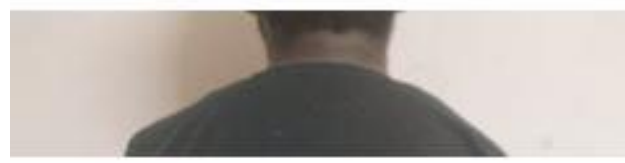

Fig. 4.4. Image Format for measurement.

\section{Finding contour \& calculating pixel length}

In order to find the contour, we are using predefined OpenCV "find contours" method on the segmented image. In order to calculate the shirt size, we need to calculate the shoulder width. And for calculation of the shoulder width, we require the images as shown in "Fig. 4.4", to acquire the extreme points effortlessly. In our case extreme points should represent the shoulder extremes. To obtain such images the user has to manually crop the image to get the desired output. To remove this manual operation we are proposing an algorithm to automate this process, making it time efficient and user-friendly. This algorithm automatically crops the image to half of its height for images taken from 75, $100 \mathrm{~cm}$ distances and finding the extreme points (red, green colour dots) shown in "Fig. 4.3". We are estimating that the extreme points will be near the upper waist region. But for the image that is taken from $150 \mathrm{~cm}$, it may not be possible if we apply the same logic. So we are taking 4/9th of the input image in order to achieve the same. Store these three readings for further calculation.

\section{Predicting shoulder size}

As we discussed earlier we are using regression analysis, a predictive modelling[9] approach to predict the actual size of the shoulder. In statistical modelling, regression analysis[10] is a set of statistical processes for estimating the relationship among variables, when the focus is on the dependent variable (Actual size of the shoulder) and one or more independent variables (calculated shoulder's pixel length of images). In this type of analysis, we need to first train the system with known values and then we can find the relationship. Here we are finding the relationship between the calculated pixel lengths and the actual size of the shoulder that has to be predicted. If we look deeper, this pixel length is dependent upon the clarity/focal length of the camera we used. To overcome this it's required to calculate the ratio of calculated pixel length to width of the image. Now we find the relationship between those ratios and the actual size of the shoulder. As we discussed, our model should be trained with known data. So collect the dataset in the form of "Table. 4.1".

Table. 4.1. Format of The Dataset

\begin{tabular}{|l|l|l|l|l|}
\hline S.no & W75 & Width75 & Ratio75 & $\begin{array}{l}\text { Actual in } \\
\text { cm }\end{array}$ \\
\hline 1 & 552.082 & 960 & 0.5750 & 47 \\
\hline 2 & 637.007 & 960 & 429.004 & 43 \\
\hline 3 & 554.008 & 960 & 433.004 & 43 \\
\hline
\end{tabular}

Here "W75" is the calculated pixel length of the extreme points, "width75" is the image width and "ratio75" is the ratio between these two variables. Similarly the other variables Width100,..,Ratio150).
(W100,

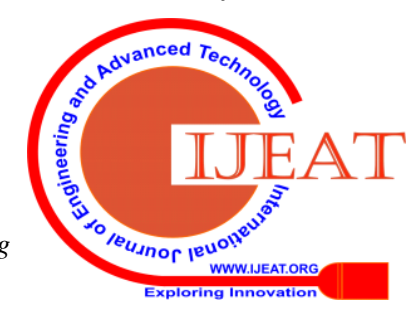




\section{Application of Image Processing In E-Commerce}

"Actual" is the actual shoulder length of the person (calculated through tape). After collecting the dataset, train the model using multi polynomial regression. This forms the relationship between actual length and the calculated variables. After training the model, there comes the prediction part. We first predicted the shirt size using only two inputs (W75, W100). In general, we decide whether our model is acceptable/good using $\mathrm{R}^{2}$ Value and mean squared error where $\mathrm{R}^{2}$ is required to be near 'one' while mean squared error is required to be as low as possible. Basically, $\mathrm{R}^{2}$ tells us how close our model is fitted to actual data and mean squared error represents the error/difference compared to actual values. Here we have done polynomial regression of degree "one" as we are getting best $\mathrm{R}^{2}(0.365)$ as shown in "Fig. 4.5" among other polynomial regression of higher degrees which is too low. Also the mean squared error was around 4.76 which isn't acceptable. Now we tried with the images given by user. We calculate the ratios of pixel length calculated and image width going through all the stages, then predicting the actual size by giving these ratios as input to our prediction model. In the last column of "Table.4.2". we can see the predicted size of the shoulder, it's almost same as the actual size. And the $\mathrm{R}^{2}$ Value comes out to be around 0.84 which is acceptable when we have done the regression with degree 'two' means not linear and shown in "Fig. 4.6", and the mean squared error is around 1.31.

\section{Scatter plot between Actual and Predicted} Values

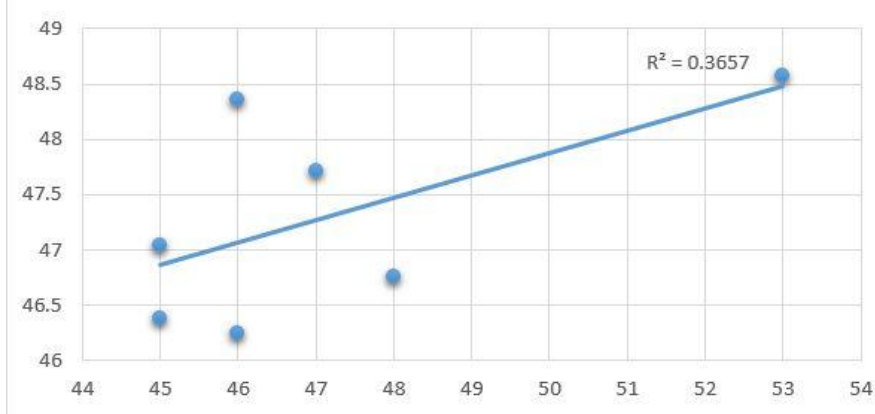

Fig. 4.5. Scatter Plot of Actual Vs Predicted values with two inputs

Table.4.2. Comparison of Actual and predicted values.

\begin{tabular}{|l|l|l|}
\hline S.no & Actual in cm & Predicted in cm \\
\hline 1 & 42 & 43.6 \\
\hline 2 & 48 & 47.8 \\
\hline 3 & 49 & 47.54 \\
\hline 4 & 49 & 48.43 \\
\hline
\end{tabular}

The above proposed system predicts the shirt size in very less time using regression model, so we can extend this technique to predict the size of different body parts which can be used to find shoe size, waist size etc. This technique can be implemented in e-commerce websites and other garment industries. If we could increase the sample size with data diverse in nature then accuracy would increase.

\section{CONCLUSION}

The proposed innovative algorithm is to measure the shirt size by giving the images as input. Firstly, Otsu binary segmentation followed by morphological operations are used for segmentation with minimum noise. Secondly, To tackle the disturbances caused by pixel capacity of camera ratio of extreme points length and the Image width is used. Later, Regression is used which made our task of predicting the shoulder width much easier and with good accuracy. Finally, the proposed algorithm achieves our objective with less speed unlike other algorithms in practice which requires learning step for contour detection also. As said, implementing this technique in e-commerce sites is one of the main applications which has the scope to increase customer satisfaction by helping customers with the correct size of their garments. 


\section{REFERENCES}

1. L. Zhao and C. Thorpe, "Recursive context reasoning for human detection and parts identification," IEEE Work. Hum. Model. Anal. Synth., no. June, pp. 136-141, 2000

2. A. Mohan, "Object Detection in Images by Components," MASSACHUSETTS Inst. Technol. Artif. Intell. Lab. Cent. Biol. Comput. Learn. Dep. BRAIN Cogn. Sci., no. 1664, 1998.

3. Y. Salih and A. S. Malik, "Depth and geometry from a single 2D image using triangulation," Proc. 2012 IEEE Int. Conf. Multimed. Expo Work. ICMEW 2012, pp. 511-515, 2012.

4. J. C. S. J. Jr, L. Dihl, C. R. Jung, M. R. Thielo, R. Keshet, and S. R. Musse, "HUMAN UPPER BODY IDENTIFICATION FROM IMAGES," Pontif ' ıcia Univ. Cat' olica do Rio Gd. do Sul , Brazil Univ. Fed. do Rio Gd. do Sul , Brazil Hewlett Packard , Brazil Isr., pp. 1717-1720, 2010.

5. J. V. Miss Hetal and P. A. Baxi, "A Review on Otsu Image Segmentation Algorithm," Int. J. Adv. Res. Comput. Eng. Technol. Vol. 2, Issue 2, Febr. 2013, vol. 25, no. 5-6, pp. 403-408, 1982.

6. Y. Zhong and B. Xu, "Automatic segmenting and measurement on scanned human body," Int. J. Cloth. Sci. Technol., vol. 18, no. 1, pp. 19-30, 2006.

7. Fisher, Perkins, Walker \&Wolfart (2003). "Spatial Filters - Laplacian of Gaussian". Retrieved 2010-09-13.

8. Nick Efford. Digital Image Processing: A Practical Introduction Using Java $^{\mathrm{TM}}$. Pearson Education, 2000.

9. F. Zahra and M. Mahmoud, "the Application of Predictive Analytics : Benefits , Challenges and How It Can Be Improved," vol. 7, no. 5, pp. 549-566, 2017.

10. I. K. Shalabh, "Chapter 3 Multiple Linear Regression Model The linear model," pp. 1-41.

\section{AUTHORS PROFILE}

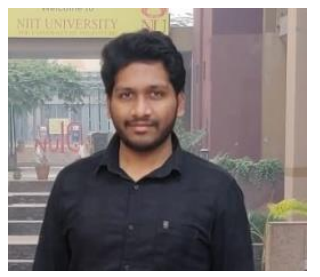

\section{LokeshVenkata Sai Mamidi}

(B Tech) UG Scholar,

Batch (2016-2020), NIIT University,

MamidiL.Sai@st.niituniversity.in

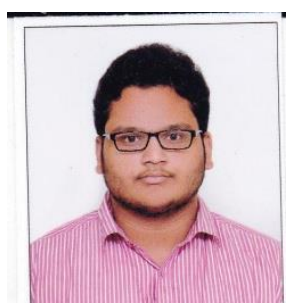

\section{Pisupati Chaitanya}

(B Tech) UG Scholar,

Batch (2016-2020), NIIT University,

pisupativ.chaitanya@st.niituniversity.in

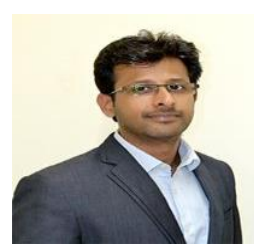

\section{VikasUpadhyaya}

Assistant Professor (ECE)

NIIT University

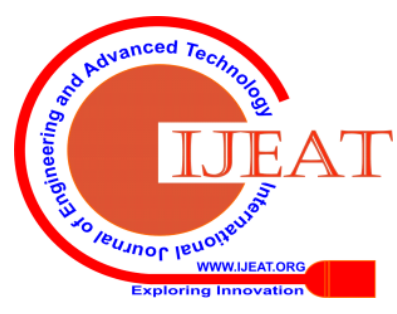

